\title{
A computerized intervention to promote colorectal cancer screening for underserved populations: Theoretical background and algorithm development
}

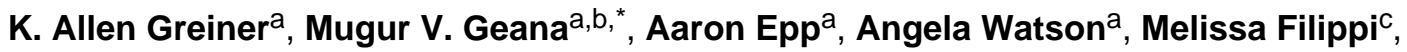 \\ Christine Makosky Daley ${ }^{c}$, Kimberly K. Engelman $^{c}$, Aimee S. James ${ }^{d}$, and Marci Campbelle \\ ${ }^{a}$ Department of Family Medicine, University of Kansas Medical Center, Kansas City, KS, USA \\ bWilliam Allen White School of Journalism and Mass Communications, University of Kansas, \\ Lawrence, KS, USA \\ 'Department of Preventive Medicine and Public Health, University of Kansas Medical Center, \\ Kansas City, KS, USA \\ dDepartment of Surgery, Washington University, St. Louis, MO, USA \\ eDepartment of Nutrition, School of Public Health, University of North Carolina Chapel Hill, NC, \\ USA
}

\section{Abstract}

Objective-The aim of this exploratory study was to assess factors deemed by patients as "important" as they planned and considered undergoing colorectal cancer (CRC) screening, and to use this data to design a computer-delivered intervention to promote screening.

\begin{abstract}
Methods-Fifty participants 50 years or older, not up-to-date with current recommended CRC screening guidelines, were recruited from a primary care clinic. A semi-structured interview focused on aspects of preparing for colorectal cancer screening was administered; after transcription, researchers used triangulation and consensus to identify relevant themes and concepts.
\end{abstract}

Results-Four main themes were identified that dealt with issues important for both FOBT and colonoscopy planning: personal concerns, reminders, communication with healthcare providers and obtaining test results. FOBT specific themes included: sample collection and return. For colonoscopy screening, themes included: scheduling, intervention questions, colonoscopy preparation, and transportation. These can be classified as barrier, process and accessory themes. The developed computer-administered implementation intentions algorithm addressed all the identified concerns in a planned and sequential manner, in order to facilitate planning for CRC screening.

\footnotetext{
(C) 2012 - IOS Press and the authors. All rights reserved

*Corresponding author: 312 Stauffer-Flint Hall, University of Kansas, Lawrence, KS 66045, USA. Tel.: +1 7858647692 ; Fax: +1 785 864 5318; geanam@ku.edu. .
} 
Conclusions-The results of this study suggest that appropriate reminders, explanations of procedures, and patient understanding of temporary life disruptions, help patients develop and accept a detailed screening plan.

\section{Keywords}

Colorectal cancer screening; FOBT; colonoscopy; implementation intentions; computer intervention; underserved populations

\section{Introduction}

Colorectal cancer (CRC) causes significant morbidity and mortality among older adults in the United States [1,2]. Available screening methods, such as colonoscopy, fecal occult blood testing (FOBT), and sigmoidoscopy, have been shown to significantly reduce mortality in average risk individuals over age fifty $[3,4]$. Although these methods have been available for several decades, Americans continue to underutilize CRC screening tests [5], especially in underserved populations [6]. To address this, interventions and programs have been developed to increase utilization of colorectal cancer screening. These have included programs focused on the health care provider $[7,8]$, the health care system $[9,10]$, the community [11], or patient directed intervention programs [7,12]. Despite some success with these interventions, rates of colon cancer screening remain just above $50 \%$ of eligible adults based on current screening guidelines [13].

Computer-based behavioral interventions conducted in public places have been shown as an effective alternative to reach underserved populations with limited access to technology $[14,15]$. Computerized health promotion and disease prevention applications allow for the intimate tailoring of the intervention to the particularities of the user [16]. The present study presents the theoretical premises and the preliminary research conducted to inform the design of an intervention algorithm for the development of a computerized application to promote colorectal cancer screening among users of safety-net clinics in the Midwestern United States.

A significant barrier to successful colorectal cancer screening is poor screening adherence, with several patient-level factors being major contributors: available resources, awareness, transportation, problems getting time off from work, discomfort with the procedures, competing priorities or cultural biases $[6,17,18]$. Although most U.S. providers recommend some type of colon cancer screening for patients over 50 years of age [19], a significant segment of patients in this group fail to obtain yearly or periodic screening. The disconnect between cancer awareness, knowledge about screening practices, physician recommendation/referral and ultimate screening completion has not been fully investigated [20]. Additional strategies may be required to ensure that patients are adequately prepared to follow-through with screening once they receive a provider recommendation or referral.

\subsection{Implementation intentions}

Emerging behavioral health theories suggests that helping patients plan for and follow through with preventive recommendations may be important for strengthening the intention- 
behavior link and thus ensuring pro-screening behaviors [21]. Implementation Intentions are an elaboration of Fishbein and Ajzen's Theory of Planned Behavior [22], and are defined as the process of thinking out the steps necessary to act on intentions toward behavior completion. This process of stating one's implementation intentions may increase confidence and strengthen intention to act [23]. Studies on papanicolaou testing and dietary change suggest that specific planning for "when" "where" and "how" patients schedule, prepare, arrange for and arrive for tests may promote behavioral completion [24-28]. However, these constructs have not been widely explored for colorectal cancer screening. Encouraging patients to identify their intended steps of when, where, and how colorectal cancer screening will be completed should increase the association between intention and action, and amplify the likelihood that screening will be completed. Nevertheless, being able to initiate and sustain discussions of when and how screening will be completed requires an in-depth understanding of how patients conceptualize and talk about specific screening steps.

\subsection{Specific aims}

This exploratory study assessed those factors deemed by patients as "important" as they described planning for and considered undergoing colorectal cancer screening. The data served as formative research for the design of a subsequent implementation intentions computer-based intervention that would-walk participants through a CRC specific implementation intentions process and would provide tailored educational materials and messages to patients in safety-net clinics. For the formative research, we aimed to assess the feasibility and acceptability of discussing CRC screening implementation steps with patients in primary care, and attempted to learn what consecutive steps toward screening completion patients identified and how they planned to implement each one; we attempted to identify critical implementation intentions language and asked participants to describe facilitators of the screening process.

\section{Methods}

\subsection{Procedures}

Participants $(N=50)$ were recruited from the primary care outpatient clinic of an academic medical institution in the Midwestern United States. Recruitment was conducted by the clinic's check out staff in cooperation with research assistants. Staff identified and approached potential participants; providing information about the study through fliers handed out to patients during the check-in process. To be eligible, participants had to be over 50 years of age, English or Spanish-speaking and self-report being not up-to-date with current colorectal cancer screening guidelines (as listed on the recruitment flyer). We created a sampling stratification system for recruitment and attempted to recruit equal numbers of men and women who were African American, Hispanic/Latino, and nonHispanic White. We utilized a purposeful sampling strategy and during recruitment asked patients about preferences for Fecal Occult Blood Test (FOBT) versus colonoscopy for screening. After providing written consent, participants were escorted to a private area of the clinic where they completed a semi-structured verbal interview focused on particular aspects of preparing for colorectal cancer screening and specific to their chosen test. Interviewers 
conducted interviews in English or Spanish, depending on patient preference, took detailed notes, and attempted to record participant responses verbatim on all questions. The study protocol was approved by the Institutional Review Board.

\subsection{Semi-structured interviews}

Questions for interviews were constructed based on implementation intention theory. Three experienced behavioral scientists reviewed and adjusted questions for readability, comprehension, and medical appropriateness. Final questions were pretested with 20 primary care patients (data not reported here); samples of final questions used in interviews are provided in Table 1.

The semi-structured interviews included questions aimed at eliciting ideas from participants about the details they believed they would need to attend to before, during, or after FOBT or colonoscopy. Interviewers described the FOBT and colonoscopy planning, preparation, and completion processes in detail and asked interviewees how they would remember each step, where and when they expected to carry the steps out, and who they anticipated asking for assistance. The questions were structured to explore participants' behavior and identify those elements that would help them create a specific plan for current or future screening actions.

Demographics and information regarding the completion of any prior colorectal cancer screening tests were abstracted from participants' medical record charts.

\subsection{Data analysis}

Interview notes were transcribed for evaluation by the research team. For interviews conducted in Spanish, two team members translated patient responses into English and reconciled any differences priorto data analysis. Qualitative analysis was conducted using an iterative process and following standard techniques [29-34]. The constant comparative technique developed by Glaser and Strauss [35] was used to determine specific coding categories.

Four team members read through all detailed interview notes, identified first level codes and summarized key findings. During analysis meetings, memos were shared and first level codes were grouped into categories through a consensus process. Each category was named with an identifiable descriptive term and definitions were produced. Team members then independently recoded interview note data using the descriptive categories. When necessary, new categories were identified and recorded in memos. These memos were shared at consecutive team meetings and original and new categories were clustered into themes.

Triangulation and consensus were used throughout the analysis phase to maximize trustworthiness of findings. Team members evaluated themes in relation to each other to develop an assessment of the relative importance of each theme. Each investigator independently assessed the salience of chosen themes by performing theme counts (total times participant comments reflected themes) and by judging the qualitative weight given to themes by participants. Team members met a final time to review theme counts and agree on relative theme importance. The entire coding and category decision-making process was 
reviewed by all team members to maximize the reliability and validity of the process and results.

Five participant's medical records later revealed that they were up-to-date with colorectal cancer screening although they reported otherwise (i.e., on review, their clinician determined new screening was not needed), data from these five individuals was retained in the qualitative data analysis.

\section{Results}

\subsection{Demographic data}

Fifty individuals completed semi-structured interviews and were included in the qualitative analysis (36 English speaking and 14 Spanish speaking). Of these individuals, 26 were female, 10 were African American and 16 were Hispanic; the mean age for participants was 60 years of age (range, 50-78). Although we had more Whites than our sampling strata called for, we were able to maintain nearly equal numbers of male and female participants.

\subsection{Implementation Intentions themes}

Under interviewer guidance, participants were willing and able to state very specific implementation intentions for following through with colorectal cancer screening. The themes that emerged from the interviews were similar between colonoscopy and FOBT, with some minor differences due to the nature of the tests.

Four main themes were identified that dealt with issues important for both FOBT and colonoscopy planning. These were; personal concerns, reminders, communication with healthcare providers and obtaining test results.

The personal concerns theme addressed personal issues (such as health issues or concerns regarding their ability to perform the test), that could influence both the test itself as well as prospective results.

For example one FOBT participant stated that:

“... okay, here is my situation with the cards: I'm red, green color blind. So if I'm looking for blood in my stool, then I've got a problem... " Another participant said:

"See, I don't have a bowel movement every day. I'm kind of slow on the bowel movements; sometimes I don't have them in a week..."

A colonoscopy participant stated that:

“... I had the test years ago and it was kind of... I was kind of frightened to have it again, because I remember before I had to take this salt solution or something, drink this salt solution. And I have hemorrhoids and I was really concerned about that again, because I remember it was such a miserable thing..."

A second theme, reminders, focused mainly on remembering to complete the tests. Although a majority stated that they would only need to make a mental note, some said that they would employ some sort of reminder to ensure they completed the FOBT cards or took the 
pre-medication for colonoscopy. Most FOBT participants addressed reminders by stating that they would place the cards in a visible and frequently visited place, such as the bathroom counter or on top of the toilet.

"I'll keep it [the cards] in the bathroom at home on the toilet" or "Umm,... I'll keep it in a position or location where I will notice them every time I have a bowel movement."

Although some participants said they would write a reminder to collect the samples, most individuals responded that they would not need other memory aids.

"I don't really have to remind myself cause I know once I get home that's what I'm going to do, just go ahead and do it and get it over with."

Colonoscopy participants described similar approaches for remembering to take the premedication, by placing it in a visible place, such as in the kitchen or bedroom:

“... I'll leave it in the kitchen on the table, so it is handy, and the instructions near my bed" or "I will keep it probably in the cabinet in the kitchen or either on the dining room table until I am ready to use it..."

The communication with healthcare providers theme addressed mainly referral, scheduling or the possibility of communicating with a provider if a problem arose while completing the test (FOBT) or during preparation for colonoscopy. Participants were not reluctant about talking with providers and the majority of them manifested a positive attitude regarding their success in obtaining the information they need:

"I'll probably just get back with the doctor [if I had problems setting up the test]" or "Well, If I couldn't remember [the directions] I would call here to Family Practice and they would put me through to the nurse..."

To obtain their test results, interviewees said they would call or ask their health care provider or their provider's staff to get the results. Most of them planned to be proactive in getting in touch with their providers to get the results:

"I'll call Dr. G..., [to learn about test results]" or "Probably by mail. Email for some people is better, but mail is usually how you get things, or a phone call."

"[I'll contact] my primary physician ... to get all my results."

"I'd have to call and ask. Or I'd expect to be notified by telephone or a follow-up appointment."

FOBT specific themes included: sample collection and sample return. As mentioned earlier, interviewees typically identified a designated location to store the FOBT kit. Participants most often described the location as a convenient place that would act as a visual reminder to collect samples (the bathroom or bedroom were the most cited examples). Those who provided further elaboration stated that the FOBT collection would remain on their minds, they were anxious about it, or that they wanted to know the results of the test, so they felt unlikely they would forget the test. Almost all participants planned to collect the samples at home rather than carrying the kit with them or doing it away from home. No one reported 
anticipating feeling embarrassed or nervous collecting samples. Generally, participants reported they believed it would take less than a week to collect the three samples.

"I'll collect the samples at home. I would be embarrassed to do it anywhere else" or

"I don't know... it depends on how my bowels will work. If my bowels work good, I can, you know, do it in a week..."

As for returning the kit once the samples were collected, participants mentioned writing notes on a calendar or having the visual cue of the FOBT kit, as potential reminders. Some participants were more creative:

"I'll tie a ribbon around my finger. When they're full I'll know to mail it [the FOBT kit]." or "I can set up events on my PDA, so the alarm will go off and remind me."

Most participants planned to send the samples immediately upon completion via mail. A small minority preferred to deliver the samples to their health care provider's office or the lab themselves.

"I'll mail the kit the same day I'm done collecting my last sample" or "I know at the third day it got to be mailed in. I don't want it sitting around."

For colonoscopy screening, the specific themes included: scheduling, intervention questions, colonoscopy preparation, and transportation.

Interviewees mostly stated they would schedule an appointment using their home phone. They described a preference for speaking to a live person for scheduling, although the majority of individuals stated they would leave a message and wait for a return phone call.

"Oh..., I would assume that I would have to talk to somebody here in the Family Practice Clinic and get a referral to a gastroenterologist."

"I don't think it will be a problem [with scheduling], but you know, I can always call back. Or leave a message. Usually within Family Practice they call you back within 24 hours."

In contrast to FOBT, many participants indicated that they believed they would have additional questions regarding the screening process, mainly addressing the administration of medication, procedure details, and recovery expectations. Overall, colonoscopy participants had more need for information on the procedure, compared to those that preferred the FOBT screening test:

"I want to know where I would be going, exactly. And how would I go about getting the medication. Would they call in to a pharmacist or do I go and pick it up, or exactly what kind of procedure would I have to go through to get the medicine that I have to take to be cleaned out?"

Worries about taking the medication and compliance also surfaced in the interviews. Many participants acknowledged a need for a family member or friend to help them with the administration of the medication and keeping them on track. For this portion of the 
screening procedure it seemed that completion, to some extent, involved a group effort - and the engagement of family, friends or others in a patient's "circle of care":

"I'll tell my husband, he'd remember. I have four children... three grown children who live here in the city and I have grandchildren, so there are people who would remind me." or

"I would tell my wife this is what I had to do." or

"Everything that we do we would probably have my wife involved in all of the activities that we do. (... ) I want her to kind of just, you know, make sure I got my clear liquids and I probably would want my pillow tucked and remote control given to me and all of that."

Most described that they believed they would pick up colonoscopy preparation medication once information or prescriptions were available or the day before administration, and probably would have to plan for it (in terms of timing). Transportation to the pharmacy or costs did not seem to be issues that required planning ahead of time.

Nevertheless, going to and from the hospital, and also dealing with absence from work or other daily activities did require preliminary planning and was noted as an important factor ensuring compliance with the screening process:

"I would make arrangements to have the youngsters taken care of and whatever obligations I would have for that time - a neighbor or I would hire someone to do that [house work]."

"I'll have to get a morning appointment because I would call Bus Respond. And I have to call it in three... three days before I get ready to come back out here. They bring you here in the morning and then they'll pick you up whenever you need to be picked up, but you have to get out of here before 2:00 pm."

A majority of participants named family members as the main source they would tap for any help, especially for transportation to and from the hospital or colonoscopy facility.

“Actually, if that don't cause any drowsiness I'll prefer I'll be driving myself, or having my daughter or her husband. Anyway, I'll have someone to bring me; family member."

"[Last time I had a colonoscopy] It was better for someone to bring me... my husband brought me to the test. He stayed here with me and then he was there when I got ready to leave."

\section{Discussion}

This study suggests that several key themes describe the specific planning and commitment process of completing CRC screening with both FOBT and colonoscopy. We found that primary care patients,even those initially unfamiliar with CRC screening tests, were willing and able, under guidance from interviewers, to describe ways they believed they would plan for and discuss all the details necessary for completion of both types of CRC screening tests. 
Although we were able to identify common planning themes as well as test-specific themes, it is worth mentioning that not all themes bear the same weight when it comes to the implementation intention process. Themes such as personal concerns, scheduling or transportation can be considered barrier themes - failure to address them in the planning process may be very crucial in noncompliance with the screening. Attention to these themes should be mobilized early in the implementation intentions process so patients have the time to identify resources or support networks that allow them to address these barriers.

Other themes such as sample collection, sample return, colonoscopy preparation were identified as process themes. Although failure to plan for these themes may not immediately influence screening outcomes, they may become serious problems that impede the expected screening process and have the potential of delaying or derailing screening; timely identification may guide patients towards additional informational or supportive resources.

A third category we identified was accessory themes. Communication with healthcare providers and obtaining test results were examples of accessory themes. These themes usually require minimal planning on the part of the patient, and may vary depending on the patient's prior experience with the health system. Nevertheless, failure to account for or accomplish them may provide patients with a degree of personal discomfort, doubt or impede future screening.

Most participants reported that planning would be helpful in promoting completion of colorectal cancer screening. Further study will be required to determine whether, implementation plans and goal setting to overcome specific logistic or perceptual barriers can facilitate the translation of intent into action in this area.

In order for the implementation intentions process to work, it is paramount for participants to have pertinent and complete information about CRC screening, so they can identify and address possible issues that may interfere with screening completion. Based on the findings from the present study, we developed a computer-administered implementation intentions algorithm that can be deployed easily on computerized kiosks in medical clinics and help patients identify problems and plan out the details of the screening process (Fig. 1).

\section{Limitations}

The major limitations include the relatively small convenience sample and the single clinical location we used for recruitment. Further studies should explore the impact of addressing implementation intentions on CRC screening, and the feasibility of a computerized system employing the developed algorithm.

In conclusion, we found that use of Implementation Intentions approaches to ensure active engagement in the planning process may increase screening test completion. Because underserved and minority groups have not experienced the same decreases in CRC mortality since the 1990s as the majority population [36], it is possible that they may require even greater emphasis on this "post recommendation" follow-through than the typical average risk patient $[6,37]$. 
A clinic-based computerized system that can deliver tailored information about CRC screening and can provide the patient with a personalized screening plan may help with patient's adherence. The results of this study suggest that appropriate reminders, explanations of procedures, and patient understanding of temporary life disruptions, help patients develop and accept a detailed screening plan. These findings serve as the basis for the computer application development that will be undergoing further testing in a randomized trial assessing the computerized intervention that uses implementations intentions as the primary intervention construct.

\section{Acknowledgments}

This study was supported by a National Institutes of Health R01 (CA123245-02).

\section{References}

[1]. Ries, LAG.; Eisner, MP.; Kosary, CL.; Hankey, BF.; Miller, BA.; Clegg, L., et al. SEER Cancer Statistics Review, 1973-1999. National Cancer Institute; Bethesda, MD: 2002.

[2]. Jemal A, Murray T, Samuels A, Ghafoor A, Ward E, Thun MJ. Cancer statistics, 2003. CA Cancer J Clin. Jan-Feb;2003 53(1):5-26. [PubMed: 12568441]

[3]. Sirovich BE, Schwartz LM, Woloshin S. Screening men for prostate and colorectal cancer in the United States: does practice reflect the evidence? Jama. Mar 19; 2003 289(11):1414-1420. [PubMed: 12636464]

[4]. Jimbo M, Meyer B, Hyslop T, Cocroft J, Turner BJ, Weinberg DS, et al. Effectiveness of complete diagnostic examination in clinical practice settings. Cancer Detect Prev. 2006; 30(6):545-551. [PubMed: 17113240]

[5]. Trends in screening for colorectal cancer-United States, 1997 and 1999. MMWR Morb Mortal Wkly Rep. 2001; 50(9):162-166. [PubMed: 11393486]

[6]. Khankari K, Eder M, Osborn CY, Makoul G, Clayman M, Skripkauskas S, et al. Improving colorectal cancer screening among the medically underserved: a pilot study within a federally qualified health center. J Gen Intern Med. Oct; 2007 22(10):1410-1414. [PubMed: 17653808]

[7]. Fitzgibbon ML, Ferreira MR, Dolan NC, Davis TC, Rademaker AW, Wolf MS, et al. Process evaluation in an intervention designed to improve rates of colorectal cancer screening in a VA medical center. Health Promot Pract. Jul; 2007 8(3):273-281. [PubMed: 17606952]

[8]. Ferreira MR, Dolan NC, Fitzgibbon ML, Davis TC, Gorby N, Ladewski L, et al. Health care provider-directed intervention to increase colorectal cancer screening among veterans: results of a randomized controlled trial. J Clin Oncol. Mar 1; 2005 23(7):1548-1554. [PubMed: 15735130]

[9]. Sheridan SL, Harris RP, Woolf SH. Shared decision making about screening and chemoprevention. a suggested approach from the U.S. Preventive Services Task Force. Am J Prev Med. Jan; 2004 26(1):56-66. [PubMed: 14700714]

[10]. Zauber AG, Lansdorp-Vogelaar I, Knudsen AB, Wilschut J, van Ballegooijen M, Kuntz KM. Evaluating Test Strategies for Colorectal Cancer Screening: A Decision Analysis for the U.S. Preventive Services Task Force. Ann Intern Med. Oct 6.2008

[11]. Weinrich SP, Weinrich MC, Stromborg MF, Boyd MD, Weiss HL. Using elderly educators to increase colorectal cancer screening. Gerontologist. Aug; 1993 33(4):491-496. [PubMed: 8375677]

[12]. Pignone M, Harris R, Kinsinger L. Videotape-based decision aid for colon cancer screening. A randomized, controlled trial. Ann Intern Med. Nov; 2000 133(10):761-769. [PubMed: 11085838]

[13]. Shapiro JA, Seeff LC, Thompson TD, Nadel MR, Klabunde CN, Vernon SW. Colorectal cancer test use from the 2005 National Health Interview Survey. Cancer Epidemiol Biomarkers Prev. Jul; 2008 17(7):1623-1630. [PubMed: 18628413] 
[14]. Kreuter MW, Black WJ, Friend LB, Booker AC, Klump P, Bobra S, et al. Use of computer kiosks for breast cancer education in five community settings. Health Education \& Behavior. 2006; 33(5):625. [PubMed: 16923835]

[15]. Suggs LS. A 10-year retrospective of research in new technologies for health communication. Journal of health communication. 2006; 11(1):61-74. [PubMed: 16546919]

[16]. Kreuter M. Tailoring health messages: Customizing communication with computer technology: Lawrence Erlbaum. 2000

[17]. Lipkus IM, Lyna PR, Rimer BK. Colorectal cancer risk perceptions and screening intentions in a minority population. J Natl Med Assoc. Oct; 2000 92(10):492-500. [PubMed: 11105730]

[18]. Hoffman-Goetz L, Breen NL, Meissner H. The Impact of Social Class on the Use of Cancer Screening within Three Racial/Ethnic Groups in the United States. Ethinicity and Disease. 1998; $8: 43-51$.

[19]. Klabunde CN, Lanier D, Nadel MR, McLeod C, Yuan G, Vernon SW. Colorectal cancer screening by primary care physicians: recommendations and practices, 2006-2007. Am J Prev Med. Jul; 2009 37(1):8-16. [PubMed: 19442479]

[20]. Klabunde CN, Meissner HI, Wooten KG, Breen N, Singleton JA. Comparing colorectal cancer screening and immunization status in older americans. Am J Prev Med. Jul; 2007 33(1):1-8. [PubMed: 17572304]

[21]. Sullivan HW, Rothman AJ. When planning is needed: Implementation intentions and attainment of approach versus avoidance health goals. Health Psychol. Jul; 2008 27(4):438-444. [PubMed: 18643001]

[22]. Ajzen I. The Theory of Planned Behavior. Organizational Behavior and Human Decision Processes. 1991; 50:179-211.

[23]. Gollwitzer PM, Schaal B. Metacognition in action: the importance of implementation intentions. Pers Soc Psychol Rev. 1998; 2(2):124-136. [PubMed: 15647140]

[24]. Armitage CJ. Evidence that implementation intentions reduce dietary fat intake: a randomized trial. Health Psychol. May; 2004 23(3):319-323. [PubMed: 15099174]

[25]. Brandstatter V, Lengfelder A, Gollwitzer PM. Implementation intentions and efficient action initiation. J Pers Soc Psychol. Nov; 2001 81(5):946-960. [PubMed: 11708569]

[26]. Gollwitzer PM, Brandstätter V. Implementation intentions and effective goal pursuit. Journal of Personality and Social Psychology. 1997; 73:186-199.

[27]. Sheeran P, Orbell S. Using implementation intentions to increase attendance for cervical cancer screening. Health Psychol. May; 2000 19(3):283-289. [PubMed: 10868773]

[28]. Sheeran P, Silverman M. Evaluation of three interventions to promote workplace health and safety: evidence for the utility of implementation intentions. Soc Sci Med. May; 2003 56(10): 2153-2163. [PubMed: 12697204]

[29]. Willms DG, Best JA, Taylor DW, Gilbert JR, Wilson DMC, Lindsay EA, et al. A systematic approach for using qualitative methods in primary prevention research. Medical Anthropology Quarterly. 1990; 4(4):391-411.

[30]. Pope, C.; Mays, N. Qualitative methods in health and health services research. In: Mays, N.; Pope, C., editors. Qualitative Research in Health Care. BMJ; London: 1996.

[31]. Basch CE. Focus group interview: an underutilized research technique for improving theory and practice in health education. Health Educ Q. 1987 Winter;14(4):411-448. [PubMed: 3319971]

[32]. Mays N, Pope C. Qualitative research in health care. Assessing quality in qualitative research. Bmj. Jan 1; 2000 320(7226):50-52. [PubMed: 10617534]

[33]. Miles, M.; Huberman, AM. Qualitative Data Analysis: An Expanded Sourcebook. Sage Publications; Thousand Oaks, CA: 1994.

[34]. Miles, MB.; Huberman, AM. Qualitative Data Analysis: a Source Book of New Methods. Sage Books; Thousand Oaks, CA: 1984

[35]. Glaser, B.; Strauss, A. The Discovery of Grounded Theory: Strategies for qualitative research: Aldine Transaction. 2007.

[36]. Edwards, BK.; Ward, E.; Kohler, BA.; Eheman, C.; Zauber, AG.; Anderson, RN., et al. Cancer. Dec 7. 2009 Annual report to the nation on the status of cancer, 1975-2006, featuring colorectal 
cancer trends and impact of interventions (risk factors, screening, and treatment) to reduce future rates.

[37]. Hawley ST, Volk RJ, Krishnamurthy P, Jibaja-Weiss M, Vernon SW, Kneuper S. Preferences for colorectal cancer screening among racially/ethnically diverse primary care patients. Med Care. Sep; 2008 46(9 Suppl 1):S10-S16. [PubMed: 18725820] 


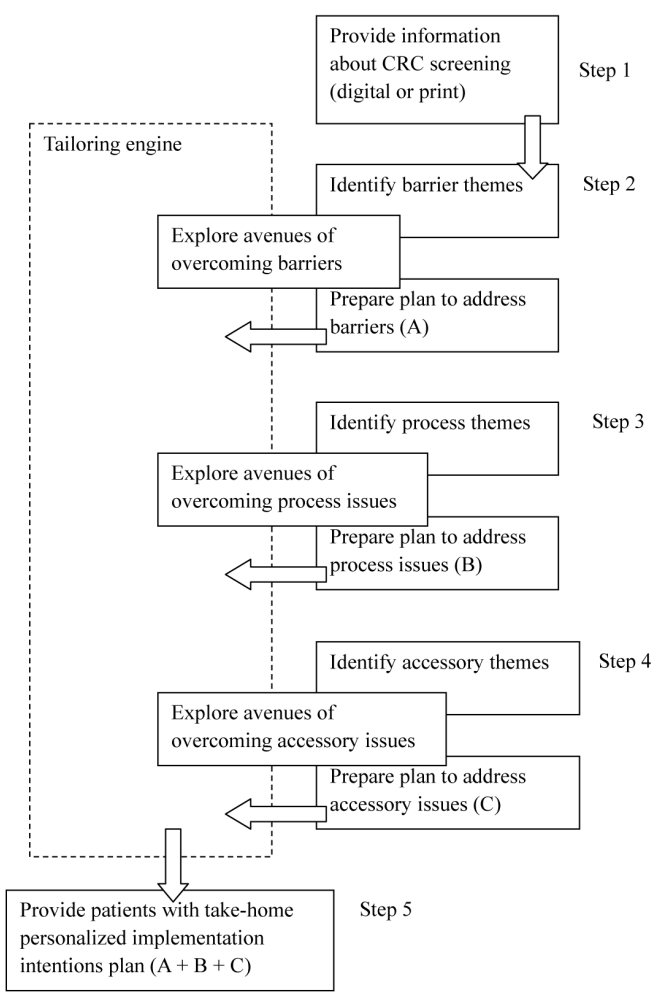

Fig. 1.

Process algorithm for implementation intentions computerized program. 


\section{Table 1}

Example questions for the semi-structured interviews

\section{FOBT example questions:}

Where will you keep your kit before you collect the samples?

What will you do to remind yourself to collect the samples?

Where do you think you will collect the samples?

When do you think you will collect your first sample (date, time of day)?

How many days do you think it will take to collect all three samples?

Where will you keep the kit between collecting samples?

How will you remember to mail in your samples?

\section{Colonoscopy example questions:}

Who will you call to set up your test for colon cancer?

When will you call to set up your test?

Where will you call from?

What phone will you use to make this call?

What will you do if you have problems setting up the test?

What questions do you want to ask when you call? 